Erratum

\title{
Erratum to "Assessment of the Levels of Level of Biomarkers of Bone Matrix Glycoproteins and Inflammatory Cytokines from Saudi Parkinson Patients"
}

\author{
Aziza Alrafiah $\mathbb{D},{ }^{1}$ Ebtisam Al-Ofi, ${ }^{2}$ Mona Talib Obaid, ${ }^{3}$ and Nimah Alsomali $\mathbb{D}^{3}$ \\ ${ }^{1}$ Department of Medical Laboratory Technology, Faculty of Applied Medical Sciences, King AbdulAziz University, \\ Jeddah, Saudi Arabia \\ ${ }^{2}$ Department of Physiology, Faculty of Medicine, King Abdulaziz University, Jeddah, Saudi Arabia \\ ${ }^{3}$ National Neuroscience Institute, King Fahad Medical City, Riyadh, Saudi Arabia
}

Correspondence should be addressed to Nimah Alsomali; nialsomali@kfmc.med.sa

Received 13 July 2020; Accepted 13 July 2020; Published 30 September 2020

Copyright (C) 2020 Aziza Alrafiah et al. This is an open access article distributed under the Creative Commons Attribution License, which permits unrestricted use, distribution, and reproduction in any medium, provided the original work is properly cited.

In the article titled "Assessment of the Levels of Level of Biomarkers of Bone Matrix Glycoproteins and Inflammatory Cytokines from Saudi Parkinson Patients" [1], multiple references were omitted in error. The references are shown below and listed as $[2,3]$.

These should supplement the following sentences in the article text:

Osteopontin (OPN) was revealed to be elaborate in inflammatory and degenerative mechanisms of the neurons [2]. OPN plays a critical role in PD due to its anti-inflammatory and antiapoptotic properties and its role in regulating iNOS transcription, reactive oxygen species production, and cytokines levels (9-10). In addition, it has been found that OPN sera and cerebrospinal fluid (CSF) amounts are greater in PD patients than controls, with CSF extent positively linked with concomitant dementia [3].

The error was introduced during the production process of the article, and Hindawi apologises for causing this error in the article.

\section{References}

[1] A. Alrafiah, E. Al-Ofi, M. T. Obaid, and N. Alsomali, "Assessment of the Levels of Level of Biomarkers of Bone Matrix Glycoproteins and Inflammatory Cytokines from Saudi Parkinson Patients," BioMed Research International, vol. 2019, no. 6, Article ID 2690205, p. 6, 2019.
[2] M. Carecchio and C. Comi, "The role of osteopontin in neurodegenerative diseases," Journal of Alzheimer's Disease, vol. 25, no. 2, pp. 179-185, 2011.

[3] W. Maetzler, D. Berg, N. Schalamberidze et al., "Osteopontin is elevated in Parkinson's disease and its absence leads to reduced neurodegeneration in the MPTP model," Neurobiology of Disease, vol. 25, no. 3, pp. 473-482, 2007. 